\title{
Gastrointestinal Ultrasound in Functional Disorders of the Gastrointestinal Tract - EFSUMB Consensus Statement
}

\section{(c) (1) $\odot$}

Authors

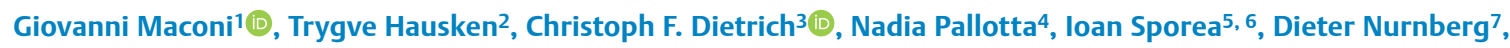
Klaus Dirks ${ }^{8}$, Laura Romanini ${ }^{9}$, Carla Serra ${ }^{10}$, Barbara Braden ${ }^{11}$, Zeno Sparchez ${ }^{12}$, Odd Helge Gilja ${ }^{13}$

Affiliations

1 Gastroenterology Unit, Department of Biomedical and Clinical Sciences, "L.Sacco" University Hospital, Milano, Italy

2 Department of Medicine, University of Bergen, Bergen, Norway

3 Department Allgemeine Innere Medizin, Kliniken Hirslanden Beau-Site, Salem und Permanence, Bern, Switzerland

4 Department of Internal Medicine and Medical Specialties, Sapienza University of Rome, Roma, Italy

5 Department of Gastroenterology, University of Medicine and Pharmacy, Victor Babes Timisoara, Timisoara, Romania

6 Department of Gastroenterology, Universitatea de Vest din Timisoara, Timisoara, Romania

7 Brandenburg Institute for Clinical Ultrasound (BICUS) - Medical University Brandenburg "Theodor Fontane", Faculty of Medicine and Philosophy and Faculty of Health Sciences Brandenburg, Neuruppin, Germany

8 Gastroenterology and Internal Medicine, Rems-Murr-Klinikum Winnenden, Winnenden, Germany

9 Department of Radiology, Radiologia 1, Hospital of Cremona, Cremona, Italy

10 Internal Medicine and Gastroenterology, University of Bologna, Hospital of Bologna Sant'Orsola-Malpighi Polyclinic, Bologna, Italy

11 Translational Gastroenterology Unit, Oxford University Hospitals NHS Foundation Trust, Oxford, UK

12 3rd Medical Department, University of Medicine and Pharmacy, Cluj-Napoca, Romania

13 Haukeland University Hospital, Haukeland University Hospital, and Department of Clinical Medicine, University of Bergen, Bergen, Norway

Keywords:

esophagus, rectum, stomach, ultrasound, small bowel

received $\quad 10.10 .2020$

accepted after revision $\quad 01.04 .2021$
Bibliography

Ultrasound Int Open 2021; 7: E14-E15

DOI $10.1055 / a-1474-8013$

ISSN 0947-7349

(C) 2021. The Author(s).

This is an open access article published by Thieme under the terms of the Creative Commons Attribution-NonDerivative-NonCommercial-License, permitting copying and reproduction so long as the original work is given appropriate credit. Contents may not be used for commecial purposes, or adapted, remixed, transformed or built upon. (https://creativecommons. org/licenses/by-nc-nd/4.0/)

Georg Thieme Verlag KG, Rüdigerstraße 14,

70469 Stuttgart, Germany

Correspondence

Giovanni Maconi

Gastroenterology Unit, Department of Biomedical and

Clinical Sciences, “L.Sacco” University Hospital,

Via GB Grassi 74

20157 Milan

Italy

Tel.: + 390239043164, Fax: + 39023942232

giovanni.maconi@unimi.it

$\circledast$

Supplementary Material is available under https://doi. org/10.1055/a-1474-8013.

\section{ABSTRACT}

Abdominal ultrasonography and intestinal ultrasonography are widely used as first diagnostic tools for investigating patients with abdominal symptoms, mainly for excluding organic diseases. However, gastrointestinal ultrasound (GIUS), as a real-time diagnostic imaging method, can also provide information on motility, flow, perfusion, peristalsis, and organ filling and emptying, with high temporal and spatial resolution. Thanks to its noninvasiveness and high repeatability, GIUS can investigate functional gastrointestinal processes and functional gastrointestinal diseases (FGID) by studying their behavior over time and their response to therapy and providing insight into their pathophysiologic mechanisms. The European Federation of Societies for Ultrasound in Medicine and Biology (EFSUMB) has established a Task Force Group consisting of GIUS experts, which developed clinical recommendations and guidelines on the role of GIUS in several acute and chronic gastrointestinal diseases. This review is dedicated to the role of GIUS in 
assisting the diagnosis of FGID and particularly in investigating patients with symptoms of functional disorders, such as dysphagia, reflux disorders, dyspepsia, abdominal pain, bloating, and altered bowel habits. The available scientific evidence of GIUS in detecting, assessing, and investigating FGID are report- ed here, while highlighting sonographic findings and its usefulness in a clinical setting, defining the actual and potential role of GIUS in the management of patients, and providing information regarding future applications and research.

\section{Introduction}

Functional gastrointestinal disorders (FGID) include several disorders involving visceral sensitivity, secretion and/or motility disturbances of various segments of the whole gastrointestinal tract.

These disorders are very common, overall affecting more than a quarter of the general population worldwide, and they represent a major medical, social, and economic burden.

Despite the fact that the diagnosis of these disorders relies mainly on clinical data, biochemical, endoscopic, and imaging investigations are often requested to exclude organic diseases.

Abdominal ultrasonography is one of the most widely used diagnostic tools for the preliminary investigation of abdominal symptoms. It is likely the easier, quicker, and cheaper noninvasive means to assess the normal and pathological biliopancreatic and gastrointestinal tract. In addition, ultrasound (US) can provide information on motility, flow, perfusion, peristalsis, and organ filling and emptying with high temporal and spatial resolution [1].

Due to its noninvasiveness and high repeatability, US scanning is useful for investigating functional processes that often have a high intraindividual variability and are sensitive to surrounding stress factors. It is therefore well suited to studying the variation with time and during therapy of the gastrointestinal tract.

Accordingly, gastrointestinal ultrasound (GIUS) may be a valuable tool in the diagnosis and management of FGID and could be of value to investigate their pathophysiologic mechanisms.

Under the auspices of the European Federation of Societies for Ultrasound in Medicine and Biology (EFSUMB), a task force group (TGF) was dedicated to investigating the role of GIUS. The creation of the TGF and the modified Delphi procedure that, relying on the data of the literature, led to the development of clinical recommendations and guidelines regarding GIUS for different specific clinical settings have been reported elsewhere [2-7]. This paper, the last of this consortium, authored by members of the TFG with scientific and clinical experience in the field of FGID who agreed to cooperate on the document, gives an overview of the role of GIUS for assessing FGID and some GI diseases with functional impairment.

Relying on the data in the literature (Supplementary file 1), statements on the usefulness of GIUS in FGID have been proposed with their Level of Evidence (LoE) and Grade of Recommendation (GoR) according to the Oxford Centre for Evidence-Based Medicine 2011. Levels of Evidence and their agreement/disagreement level have been scored on a five-point Likert scale as follows: A + : agree; A-: rather agree; I: indecisive; D-: rather disagree; D+ : disagree. The statements in the manuscript were voted on by the whole TFG.

The goal of the review is to show and discuss the scientific evidence regarding GIUS for detecting, assessing, and investigating functional disorders of the gastrointestinal tract, while highlight- ing their sonographic findings and the usefulness of the method in a clinical setting.

\section{Functional Diseases of the Esophagus}

Functional disorders of the esophagus include several conditions presenting with symptoms like dysphagia, globus, chest pain, heartburn that involve the tongue and oropharynx, cervical and distal esophagus.

Ultrasound of the oropharynx and cervical esophagus can be useful in patients with dysphagia and pharyngeal globus. Dysphagia is a common symptom (up to $13 \%$ of the population aged $>65$ years and $>50 \%$ of patients after a stroke and in residential care) [8], caused by structural or functional abnormalities of the tongue, oropharynx, or cervical esophagus (including swallowing disorders), or the distal esophagus, at the esophagogastric junction. These anatomical segments can be assessed by GIUS. In particular, the cervical esophagus is detectable to the left of the trachea, starting from the left lower pole of the thyroid. It shows a tubular structure with wall thickening $<3 \mathrm{~mm}$ and the typical layer structure. In addition, GIUS can assess esophageal peristalsis after swallowing. The distal $4-5 \mathrm{~cm}$ of the esophagus and esophagogastric junction, at the level of the diaphragm, can usually be visualized from the epigastrium using the left liver lobe as an acoustic window and tilting the transducer cranially while the patient is asked to perform a deep inspiration breath hold. In this transducer position, with a longitudinal orientation, the esophagus and cardia can be visualized ventral to the aorta, below the diaphragm.

\section{Swallowing disorders}

In patients with dysphagia, GIUS may assess the integrity and movements of the tongue and muscles during the oropharyngeal phase of swallowing. This has been used as visual feedback in dysphagia rehabilitation following partial glossectomy for cancer and stroke [9]. By placing the probe in a longitudinal axis under the chin, US allows real-time observation of the swallowing process including food propulsion by measuring the distance between the shades created from the mandible and the hyoid bone at rest and during swallowing ( $\triangleright$ Fig. 1). The difference in the distance, defined as hyoid bone displacement, correlates inversely with the amount of pharyngeal residue, and a hyoid bone displacement $<13.5 \mathrm{~mm}$ has been observed as an accurate cut-off for detecting penetration or aspiration (sensitivity $83.9 \%$, specificity $81.0 \%$ ) [10]. The anterior movement of the hyoid bone (reduced distance to the mandibula) and the larynx-to-hyoid approximation are therefore important parameters to describe the initiation of the larynx elevation and the down-folding of the epiglottis for the airway protection. 


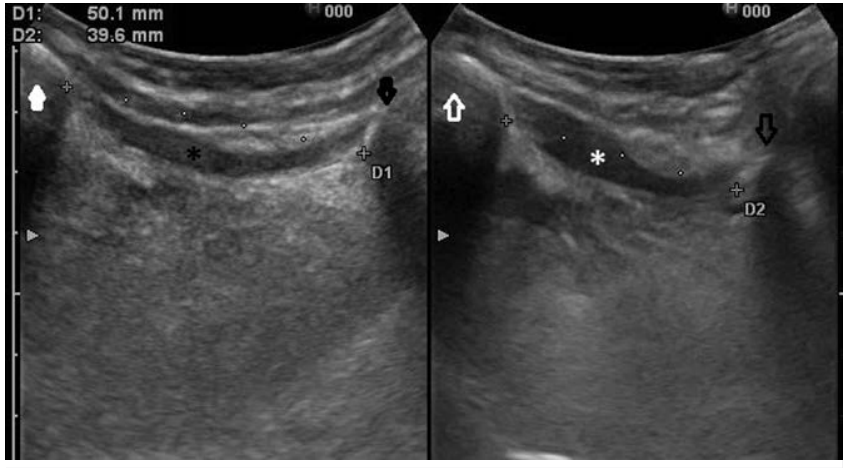

- Fig. 1 Hyoid bone displacement during swallowing. Distance between the mandible (white arrow) and the hyoid bone (black arrow) at rest (left panel) and during swallowing (right panel). Asterisk: suprahyoid muscle.

\section{Statement 1}

GIUS can be used to investigate swallowing disorders and to assist as visual feedback in dysphagia rehabilitation [LoE 4; GoR: C]

Consensus level of agreement: A+ 7/11, A-4/11 (1 abstention)

\section{Zenker's diverticulum}

Sonography of the cervical esophagus may reveal some dysphagia-correlated diseases such as Zenker's diverticulum. Zenker's diverticulum appears as a pharyngeal pouch-shaped lesion in the posterior part of the pharyngo-esophageal junction [11]. In the majority of cases, a mass with heterogeneous internal echoes is seen posteriorly, but separate from the left thyroid lobe. The mass is surrounded by a multi-layered wall and abuts the esophagus. The internal air echoes can mimic calcifications ( $\triangleright$ Fig. 2). When a suspected lesion is detected, the patient is asked to swallow water, or a US contrast agent, which changes the shape and internal echoes of the mass [12].

\section{Scleroderma}

In a portion of patients with scleroderma, GIUS can detect significant thickening of the walls with enlargement of the submucosa and proper muscle layers, and retention of a liquid bolus after swallowing, especially in patients with hypomotility of the cervical esophagus and dysphagia, in whom incomplete or feeble peristalsis is also detectable $[13,14]$.

\section{Globus}

Globus is a persistent or intermittent, non-painful sensation of a lump or foreign body in the throat experienced between meals. The clinical history and physical examination guide the diagnosis, but concerns about malignancy usually lead to unnecessary investigations. It has been shown that patients with globus have a significantly higher prevalence of thyroid nodules, especially if larger than $3 \mathrm{~cm}$ and located anterior to the trachea in an otherwise normal gland [15]. However, data from the Finnish Cancer Registry revealed that neck US showed no additional benefit of evaluating the globus etiology [16] and the Canadian Head and Neck Surgical On-

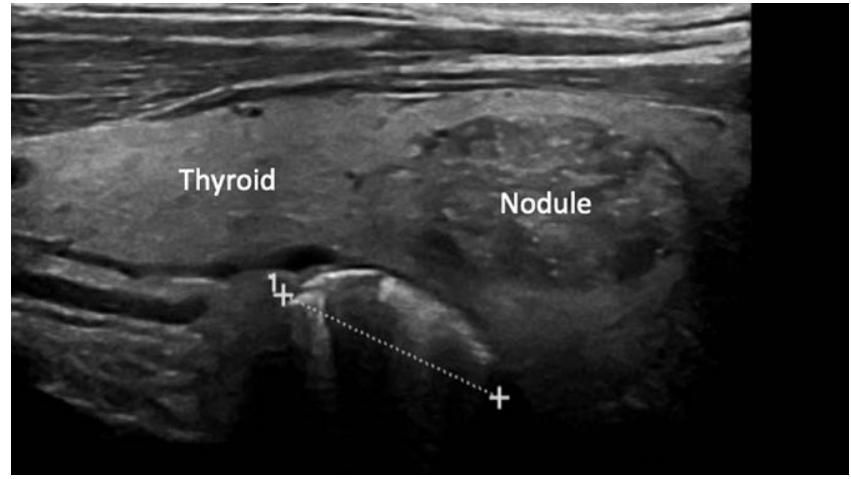

- Fig. 2 Ultrasonogram showing a sagittal section of the neck with the thyroid gland including a nodule in the front. Behind the thyroid, the esophageal tube leads to a Zenker's diverticulum demarcated between two plusses. The diverticulum is filled with air and debris.

cology Choosing Wisely Campaign does not recommend neck US to investigate globus sensation [17].

\section{Achalasia and non-cardiac chest pain}

Non-cardiac chest pain (NCCP) is a recurrent, angina-like, retrosternal chest pain of non-cardiac origin, mainly caused by Gastroesophageal reflux disease (GERD) and esophageal dysmotility. Transabdominal GIUS does not play a significant diagnostic role in this clinical context. However, it has been reported to be of some help in detecting achalasia, which is suspected when regular thickening of the muscularis propria of the esophageal wall, narrowing at the esophago-gastric junction, and dilatation of the distal esophagus with fluid retention are found [18] (> Fig. 3).

\section{Gastroesophageal reflux disease (GERD)}

The sonographic assessment of GERD relies on the evaluation of the esophagogastric junction and its real-time function. GIUS has been proposed as a noninvasive diagnostic tool in infants and children with suspected GERD, with several different diagnostic criteria, mainly the number of reflux episodes after the ingestion of a meal in a defined period of time, which may vary according to the severity of the disease (e.g., > 6 reflux episodes in $10 \mathrm{~min}$, for severe GERD) [19, 20]. A comprehensive literature review, including 11 studies, showed that GIUS has an overall high sensitivity (87\%) and moderate specificity (63\%) in detecting GERD using 24-h pH monitoring as gold standard, with increased sensitivity using color Doppler sonography [20]. On the contrary, the role of GIUS in adults is very limited. However, GIUS may detect hiatal hernia at the level of distal esophagus and esophagogastric junction (see above). Data from the literature show that patients with hiatal hernia have esophageal hiatal diameters measured via GIUS that are significantly greater than controls and that the diameter at the junction $>16 \mathrm{~mm}$ may have a positive predictive value of $>97 \%$ for detecting hiatal hernia [21].

\section{Statement 2}

Ultrasound may be a supportive diagnostic method in the assessment of functional disorders of the esophagus and cannot replace 


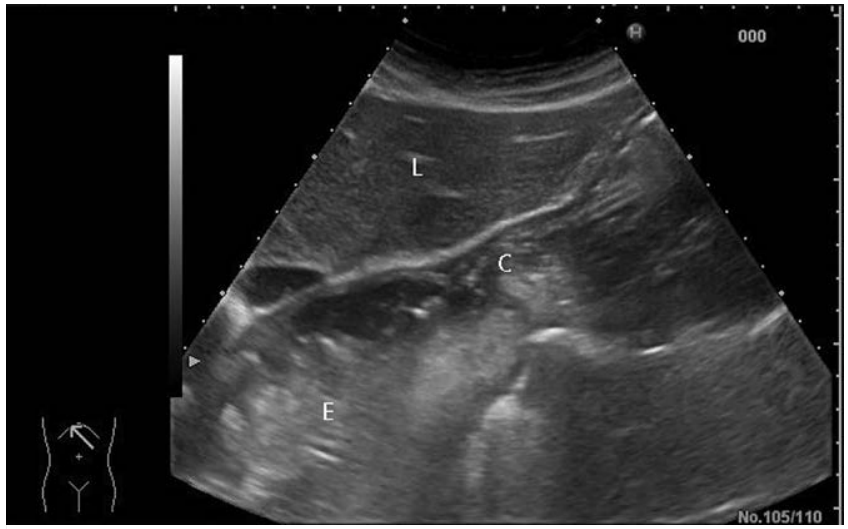

- Fig. 3 A sagittal section of the liver (L), esophagus (E), and cardia (C) of the stomach. The esophagus is markedly dilated and filled with food remnants and fluid, as typically seen in achalasia.

endoscopy, $\mathrm{pH}$-impedance, and high-resolution manometry [LoE 4; GoR: C].

Consensus level of agreement: A + 9/12, A- 3/12

\section{Statement 3}

Specific morphologic and functional sonographic findings may suggest the diagnosis of esophageal diseases when ultrasound is requested in patients with esophageal symptoms [LoE 5; GoR: C]

Consensus level of agreement: A+ 6/12, A- 6/12

\section{Dyspepsia and Other Functional Gastroduodenal Disorders}

Dyspepsia is a common disorder, affecting up to $20 \%$ of the general population. It includes 2 main categories: organic and functional dyspepsia (FD). Organic dyspepsia is mainly due to peptic ulcer, gastric cancer, pancreatic or biliary disorders. Functional dyspepsia is caused by various pathophysiological mechanisms such as delayed gastric emptying, altered antroduodenal-jejunal motility and gastric electrical rhythm, impaired gastric accommodation of a meal, hypersensitivity to gastric distension, and altered duodenal sensitivity to lipids or acids.

The diagnostic evaluation of FD may include specific second-level testing, including gastric emptying testing and examinations aimed at assessing gastric accommodation and antroduodenal motility.

Ultrasound can be used to evaluate many parameters of gastric function such as: antral contractility, gastric emptying, transpyloric flow, gastric accommodation, and intragastric distribution (volumes) of meals. The visualization and assessment of these is now supported by advanced methods such as 3D ultrasound and strain rate imaging [22, 23].

\section{Contractility of the distal stomach}

Gastric contractility and propagation of motor events can be visualized and measured by US ( $\triangleright$ Fig. 4, $>$ Video 1 ) [24]. Ultrasound enables calculation of the frequency and amplitude of contractions,

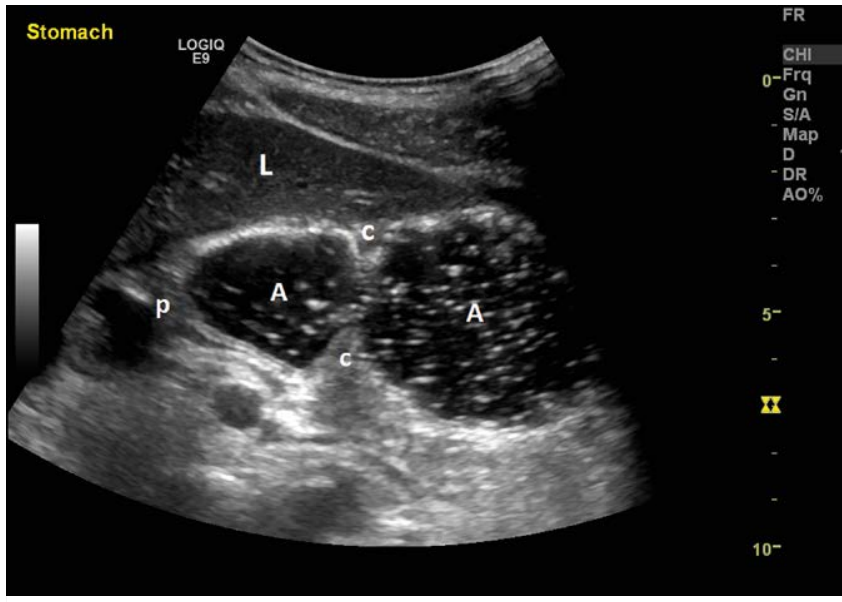

Fig. 4 Posterior to the left lobe of the liver (L), the fluid-filled stomach can be observed with some air bubbles in the lumen (A: antrum). In the middle of the antrum, a contraction (C) is making a bilateral indentation in the gastric wall, thus propelling the gastric contents towards the pylorus ( $\mathrm{p}$ ).

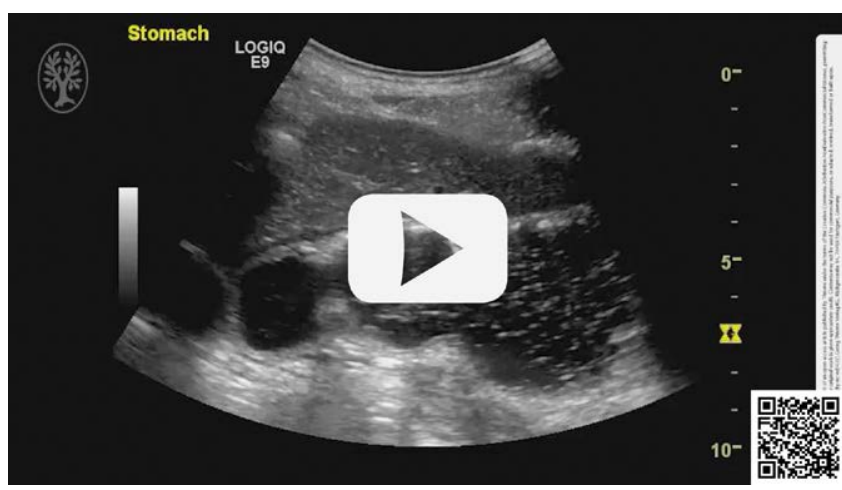

- Video 1 Horizsontal scan of the distal stomach containing a meal of soup meal in a patient with dyspepsia using the left lobe of the liver as an acoustic window. The first contraction to the left in the video (distal antrum) shows a lumen-occlusive contraction. The second wave of contractions demonstrates a non-lumen- occlusive contraction of the antrum, which would not be recorded by manometry.

with the latter being defined as the maximal reduction of antral area induced by a contraction, expressed as a fraction of the relaxed area. Two studies that investigated the relationship between ultrasonographic patterns of antral contraction, manometric pressure waves, and transpyloric fluid transport during antral phase II in healthy subjects showed that GIUS is more sensitive than manometry in detecting antral contractions, particularly non-occlusive contractions [25-27]. Acute mental stress reduces the amplitudes of antral contractions in healthy persons [28], while antral dysmotility, namely a wide range of antral excursion (the difference between circumference of the relaxed and contracted antrum), was found in patients with FD [29]. To further characterize antral contractility, ultrasound Doppler strain rate imaging was used with high accuracy with respect to assessing strain measurements in vitro [30]. In patients with epigastric pain syndrome of FD, higher 
strain levels were detected in the antrum compared to patients with postprandial distress syndrome [31].

\section{Statement 4}

Ultrasound can be used to study contractions of the stomach and can be applied to assess gastric dysmotility in patients with dyspepsia [LoE 3; GoR4].

Consensus level of agreement: A + 9/12, A- 2/12, I 1/12

\section{Gastric emptying}

Ultrasonography has been widely used to assess gastric emptying rates [32], mainly using serial measurements of the antrum area in a sagittal section, using the aorta and superior mesenteric vein as landmarks, measured before and after ingestion of a test meal ( $\triangleright$ Fig. 5). This technique showed good correlation to radionuclide estimates of emptying rates [33] both for liquid and solid meals. The estimate of gastric emptying by GIUS has been found to be delayed in $30 \%$ of patients with FD [34] and in diabetic gastroparesis [35]. Furthermore, GIUS assessment of gastric emptying has been successfully used to monitor pharmacological interventions to modify gastric emptying and to evaluate specific clinical conditions associated with delayed gastric emptying such as cirrhosis, GERD, cystic fibrosis, and systemic scleroderma.

\section{Statement 5}

Gastric emptying can be measured by ultrasonography and shows good correlation to scintigraphy. [LoE 3, GoR 4]

Consensus level of agreement: A+9/12, A-3/12

\section{Statement 6}

Ultrasound can be used to assess gastric emptying in patients with FD, gastroparesis, and other conditions in which delayed gastric emptying is suspected. [LoE 3, GoR 4]

Consensus level of agreement: A+11/12, A- 1/12

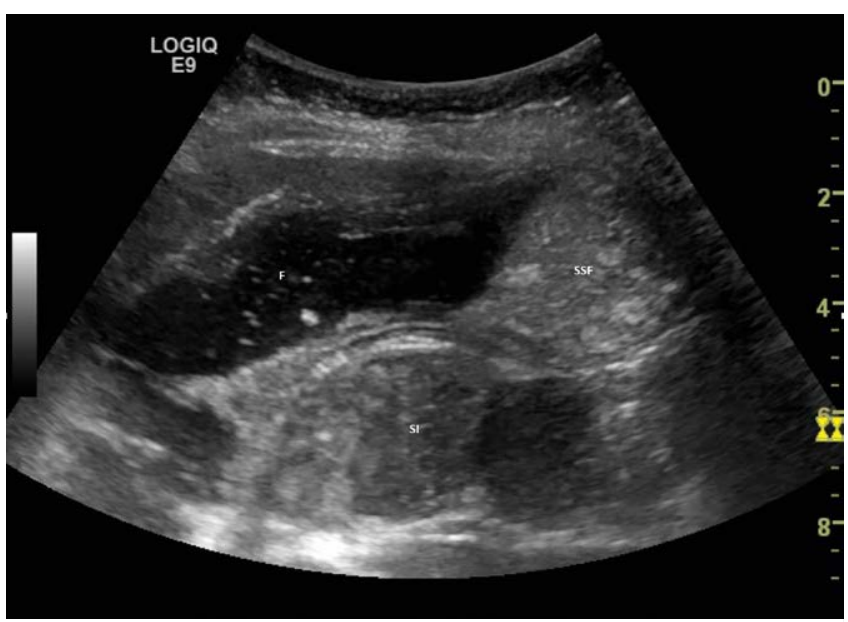

- Fig. 5 Transverse scan of the distal stomach in a patient with dyspepsia and retention of semi-solid food (SSF) shown in the right part of the gastric lumen. The stomach is predominantly filled with fluid $(F)$ shown in the left part of the gastric lumen. Posterior to the stomach, one can observe food- and fluid-filled loops of the small intestine (SI).

\section{Transpyloric flow}

The flow of gastroduodenal contents across the pylorus can be visualized by GIUS using color and spectral Doppler methods, even 3D-US, with high temporal and spatial resolution ( $>$ Fig. 6, \Video 2) [36]. Typically, an episode of flow across the pylorus has a mean velocity $>10 \mathrm{~cm} / \mathrm{s}$ lasting more than 1 second and can also happen during non-occluding peristalsis-related activity. It is associated with gastric tone of the proximal stomach and pyloric tone. Despite the fact that US evaluation of transpyloric flow is far from the routine application in clinical practice, it can provide insight into the physiology of the stomach and the pathophysiology of functional dyspepsia and can monitor pharmacological intervention for research purposes.

\section{Statement 7}

Ultrasound with and without Doppler methods can be used to visualize and calculate transpyloric flow in expert hands. [LoE 4; GoR 4]

Consensus level of agreement: A+9/12, A-3/12

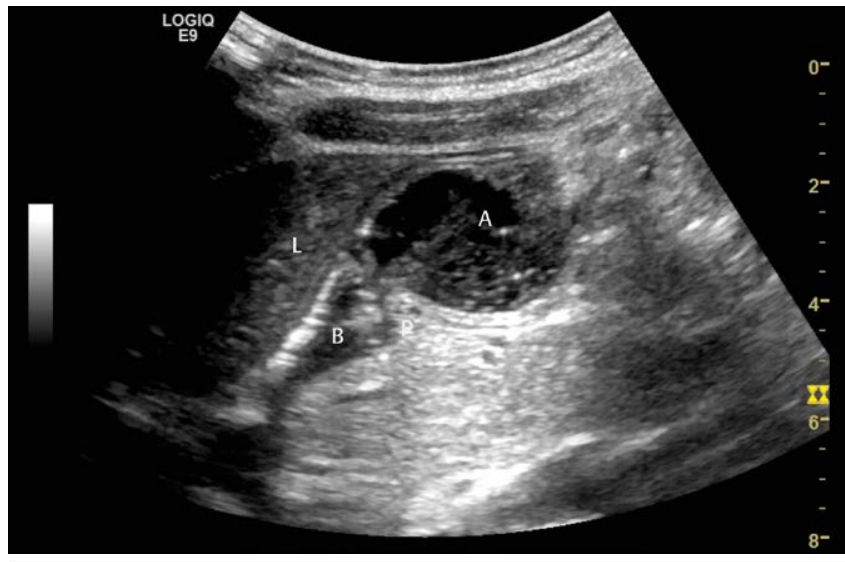

- Fig. 6 Ultrasound image of transpyloric flow demonstrating the antrum ( $\mathrm{A}$, to the right), the pylorus ( $\mathrm{p}$, in the middle), and the triangular duodenal bulb (B, to the left). The liver $(\mathrm{L})$ is shown to the far left and the pancreas posterior to the gastroduodenal segment.

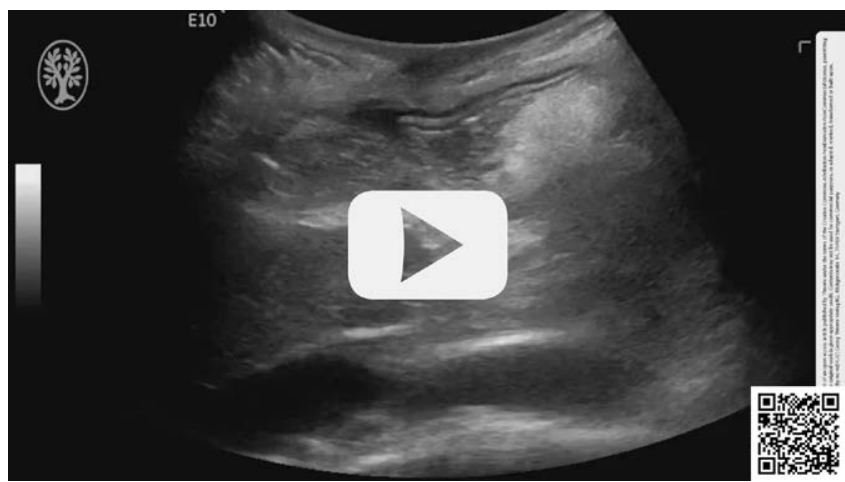

- Video 2 Ultrasound image of transpyloric flow showing the antrum (to the right), the pylorus (in the middle), and the duodenal bulb (to the left in the video). The bright content of the antrum is propelled through the pylorus as a jet into the duodenum while the antral smooth muscles are contracting, giving rise to a thickened muscularis propria of the prepyloric segment. 


\section{Accommodation of the proximal stomach}

Gastric accommodation is a key reflex to temporarily store ingested food and to control its release into the distal stomach and the small intestine. It consists of a reduction in gastric tone and an increase in compliance in response to food intake. This allows for increased volume of the proximal stomach without a corresponding rise in intragastric pressure. This process involves both intra- and extragastric reflexes and has two components: passive meal distension of the gastric compartments and active muscle relaxation of the gastric wall. The first component is best measured with imaging methods like US, whereas the latter is best assessed by a barostat, which is positioned inside the stomach via a pharyngeal route [37]. The sonographic method to evaluate gastric accommodation relies on measurements of a sagittal area and an oblique frontal diameter of the fundus as well as the antral area before and after a standard meal ( $\vee$ Fig. 7). The US assessment of proximal gastric size to estimate accommodation of meals has been shown to be accurate compared to the gold standard and useful to assess impaired gastric accommodation in patients with FD [38]. Impaired gastric accommodation after a standard meal has been shown in various studies between $30-60 \%$ of patients with FD [39].

Clinical applications of 2D US methods for estimating the size of the proximal stomach [40] have been used to study gastric accommodation in diabetes mellitus, reflux esophagitis, and recurrent abdominal pain in children, and to assess pharmacological interventions (Suppl 1).

\section{Statement 8}

Ultrasound can be used to noninvasively evaluate gastric accommodation of liquid meals. [LoE 3, GoR 4]

Consensus level of agreement: A + 8/11, A-3/11 (1 abstention)

\section{Gastric volumes and 3D ultrasound}

To improve volume estimation of intragastric contents and volumes of the gastric compartments, a method based on mechanical tilting acquisition of three-dimensional (3D) ultrasonography was developed, its accuracy and reliability were verified, and it was then applied in patients with FD [41]. Subsequently, the acquisition of 3D images was improved by using a magnetometer-based position and

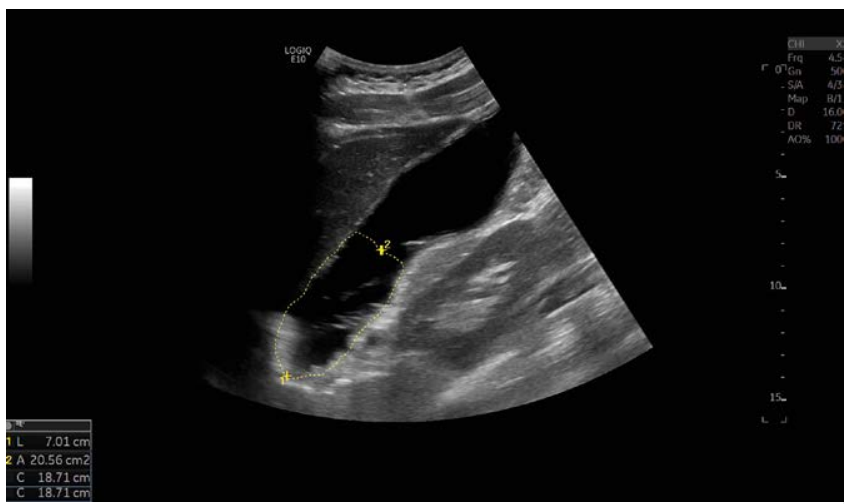

- Fig. 7 A standardized section of the fluid-filled proximal stomach with tracing of an area indicating the size of gastric accommodation. The liver is shown to the left and the left kidney to the right in the image. orientation measurement device to allow the estimation of a large organ volume like the fluid-filled stomach ( $>$ Fig. 8) [41]. These methods and others based on commercially available 3D probes with built-in tilting capacity and automated volume acquisition were used to study gastric volumes and intragastric distribution of meals [42]. $3 \mathrm{D}$ ultrasound in general is often performed with the transducer held stationary in a single position using a motorized probe device for acquisition. However, this method does not usually allow visualization of the total gastric volume. Accordingly, a magnetic scanning concept was developed to enable 3D scanning of the whole gastric compartment in one sweep starting at the proximal end and moving the probe towards the pyloric region [42].

\section{Statement 9}

3D ultrasound can be used to accurately estimate intragastric volumes and intragastric distribution of meals [LoE 3, GoR 4].

Consensus level of agreement: A+ 6/11, A- 5/12 (1 abstention)

\section{Ultrasound meal accommodation test}

Ultrasonography can estimate most parameters of gastric motility including gastric configuration and strain of the gastric wall [43, 30,44 ] and is well suited to study the effect of a standardized meal on gastric function and symptoms in patients with dyspepsia. One approach is to use a low-caloric soup meal $(20 \mathrm{kcal})$ in a standard volume $(500 \mathrm{ml})$ consumed in a specified time ( $4 \mathrm{~min})$ in combination with US scanning of the proximal and distal stomach before and after the meal with symptom registration (

\section{Statement 10}

A standardized ultrasound meal accommodation test can characterize motility disturbances in patients with FD [LoE 4; GoR 4]

Consensus level of agreement: A + 10/11, A-1/11 (1 abstention)

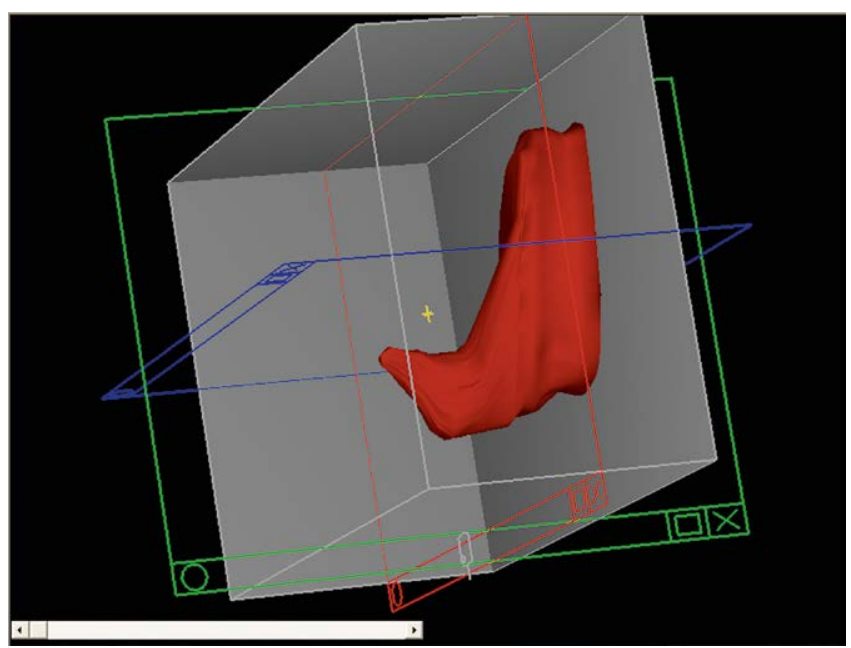

- Fig. 8 3D image reconstruction of the fluid-filled stomach based on ultrasound acquisition with a magneto-based position and orientation measurement system (POM) using GE Logiq 9. The 3D image is shown inside the frames of the three orthogonal planes used for orientation. 


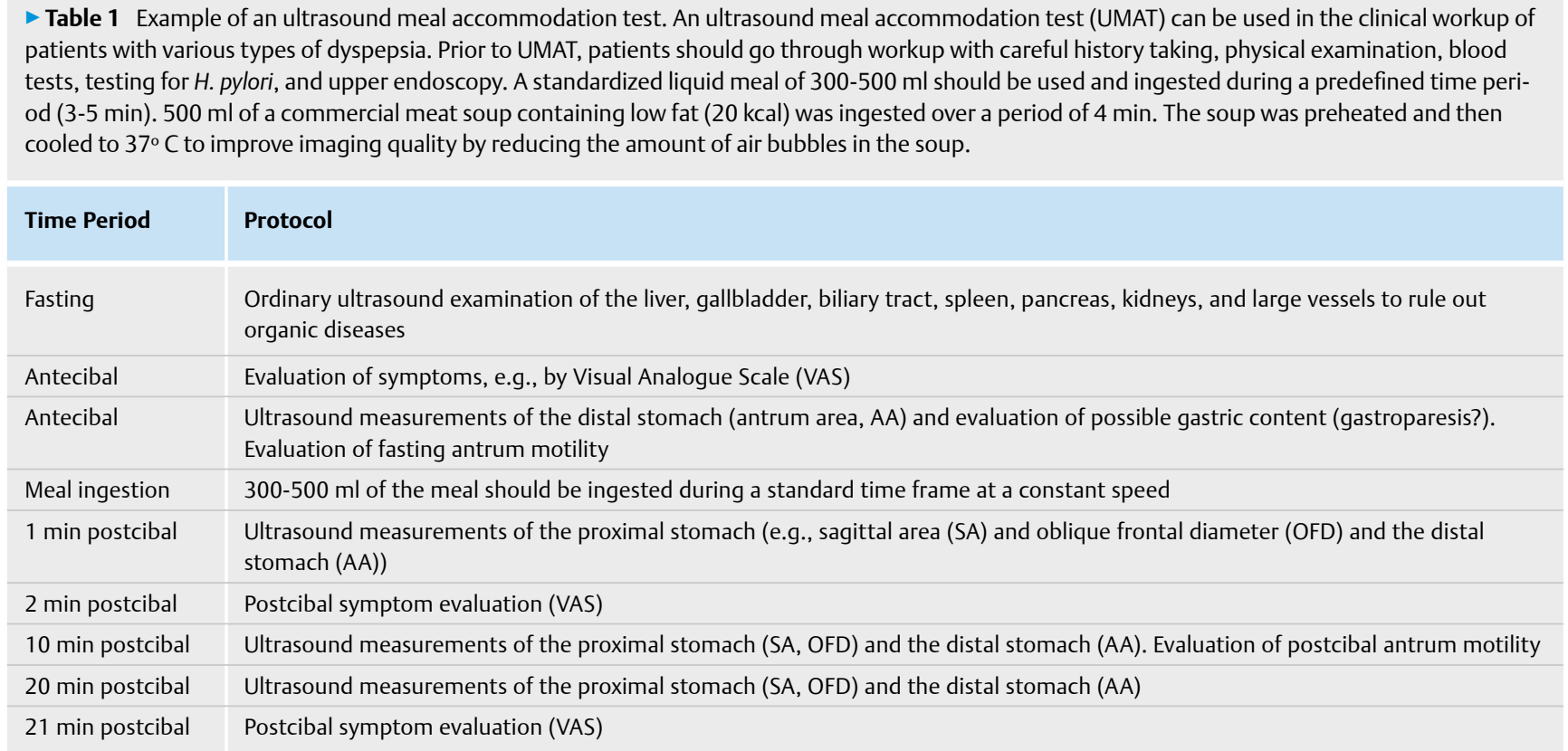
patients with various types of dyspepsia. Prior to UMAT, patients should go through workup with careful history taking, physical examination, blood tests, testing for $\mathrm{H}$. pylori, and upper endoscopy. A standardized liquid meal of 300-500 ml should be used and ingested during a predefined time period (3-5 min). $500 \mathrm{ml}$ of a commercial meat soup containing low fat ( $20 \mathrm{kcal})$ was ingested over a period of $4 \mathrm{~min}$. The soup was preheated and then cooled to $37^{\circ} \mathrm{C}$ to improve imaging quality by reducing the amount of air bubbles in the soup.

\begin{tabular}{|c|c|}
\hline Time Period & Protocol \\
\hline Fasting & $\begin{array}{l}\text { Ordinary ultrasound examination of the liver, gallbladder, biliary tract, spleen, pancreas, kidneys, and large vessels to rule out } \\
\text { organic diseases }\end{array}$ \\
\hline Antecibal & Evaluation of symptoms, e.g., by Visual Analogue Scale (VAS) \\
\hline Antecibal & $\begin{array}{l}\text { Ultrasound measurements of the distal stomach (antrum area, AA) and evaluation of possible gastric content (gastroparesis?). } \\
\text { Evaluation of fasting antrum motility }\end{array}$ \\
\hline Meal ingestion & $300-500 \mathrm{ml}$ of the meal should be ingested during a standard time frame at a constant speed \\
\hline 1 min postcibal & $\begin{array}{l}\text { Ultrasound measurements of the proximal stomach (e.g., sagittal area (SA) and oblique frontal diameter (OFD) and the distal } \\
\text { stomach (AA)) }\end{array}$ \\
\hline 2 min postcibal & Postcibal symptom evaluation (VAS) \\
\hline 10 min postcibal & Ultrasound measurements of the proximal stomach (SA, OFD) and the distal stomach (AA). Evaluation of postcibal antrum motility \\
\hline 20 min postcibal & Ultrasound measurements of the proximal stomach (SA, OFD) and the distal stomach (AA) \\
\hline 21 min postcibal & Postcibal symptom evaluation (VAS) \\
\hline
\end{tabular}

If there are signs of delayed gastric emptying after $20 \mathrm{~min}$, further ultrasound examinations can be continued, e.g., 30 and 40 min postcibal. Psychometric evaluation (questionnaires) can be added and performed on demand. Finally, the doctor concludes the UMAT session by informing the patient of the test results and providing advice regarding therapeutic options.

\section{Functional Abnormalities of the Small Bowel and Colon}

\section{Irritable bowel syndrome (IBS)}

Along with colonoscopy, abdominal US is the most frequently performed examination in patients with abdominal complaints and is requested in more than $50 \%$ of IBS patients, irrespective of the kind of IBS $[45,46]$.

Despite not being included in most IBS management guidelines, abdominal US is highly requested because it is noninvasive, cheap, widely available, and useful to rule out organic diseases. Considering that abdominal US usually does not include assessment of the bowel, the requests for US could increase in the future if GIUS were to become part of or be added to general abdominal US, in particular if coupled with biochemical tests, for excluding IBD [47]. To date, GIUS is used in patients with abdominal symptoms primarily to discriminate IBS from organic diseases, especially in patients with abdominal pain and diarrhea because it can reveal thickening of bowel walls, enlarged mesenteric lymph nodes, dilation of the small bowel, or findings suggestive of IBD and Crohn's disease ( $\mathbf{F i g . 9}$ ) as well as other less common conditions [4, 7, 48]. Unfortunately, GIUS like other imaging techniques [49] does not provide specific features of IBS. Although increased thickness of the muscularis propria of the sigmoid colon has been reported in women with IBS [50], GIUS can mainly provide and corroborate an exclusion diagnosis of IBS.

\section{Small intestine bacterial overgrowth}

Small intestine bacterial overgrowth (SIBO) is a form of intestinal dysbiosis associated with intestinal motility disorders. The most common symptoms are diarrhea, flatulence, abdominal pain, and bloating. To date, the role of GIUS has never been investigated in this context. However, a retrospective study showed that SIBO was correlated with an increased amount of fluid in the small bowel, thickened jejunal and small-bowel folds, an increased amount of gas in the intestine, and increased peristalsis in more than $84 \%$ of patients [51].

\section{Familial GUCY2C diarrhea syndrome}

Familial GUCY2C diarrhea syndrome is a rare, autosomal dominant inherited disease characterized by chronic diarrhea without significant abdominal pain, sometimes associated with Crohn's disease and small bowel obstruction and pseudo-obstruction. GIUS may show increased fluid in the bowel with turbulent intraluminal and back and forth flow of contents without propulsive movements, and a high proportion of non-occlusive contractions (

\section{Statement 11}

Specific GIUS features of IBS are not established, but the use of GIUS in patients with symptoms mimicking IBS can be of value to discriminate this entity from organic diseases [LoE 4, GoR 4].

Consensus level of agreement: A + 11/12, A- 1/12

\section{Symptomatic uncomplicated diverticular disease of the colon (SUDD)}

SUDD is a clinical entity including colonic diverticulosis associated with persistent abdominal pain, bloating, and changes in bowel habits but not associated with overt inflammation. Currently there is not a universal gold standard in the diagnosis of this condition. Colonoscopy and CT colonography play a pivotal role in detecting 

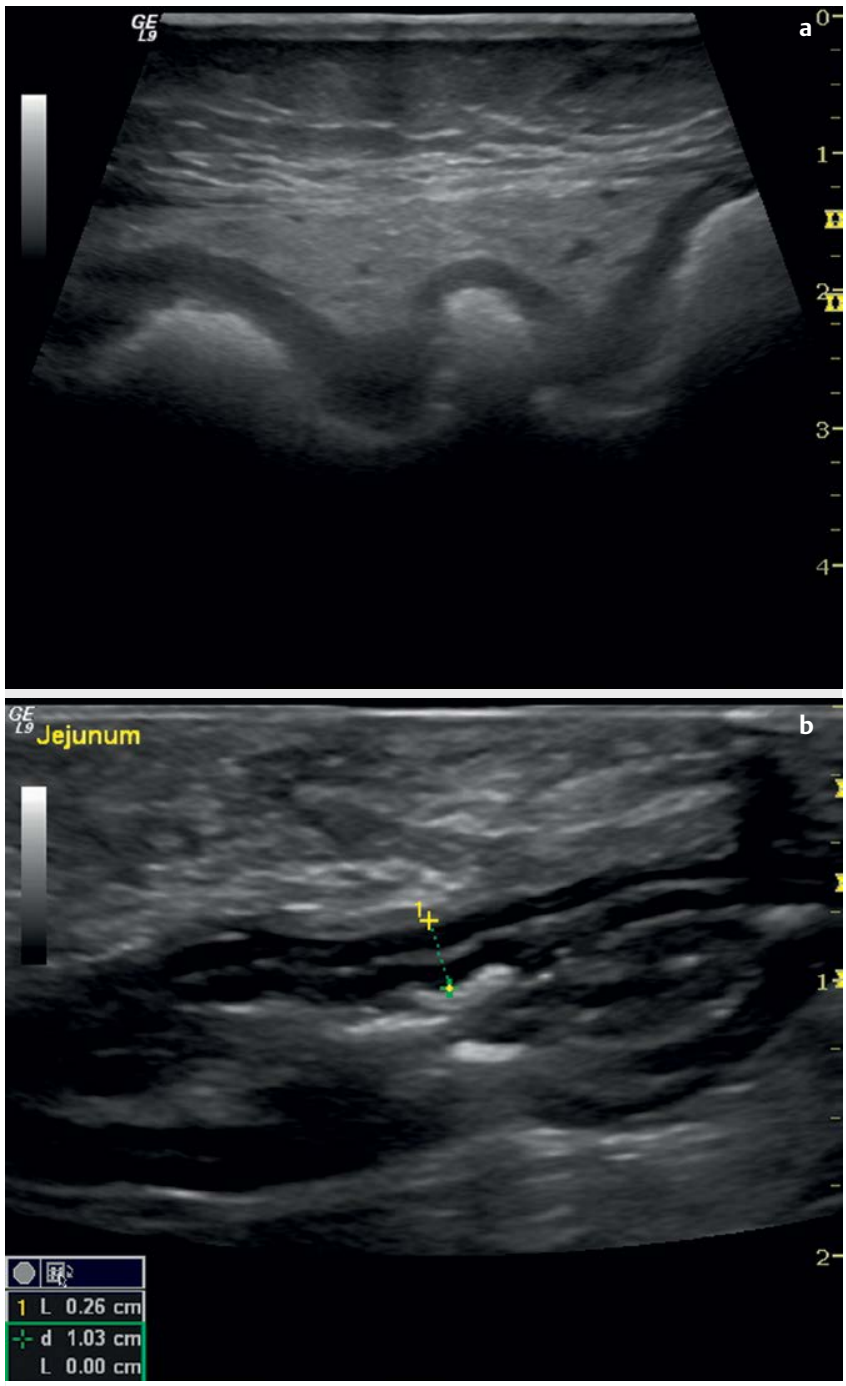

- Fig. 9 a Ultrasonogram showing a segment of the descending colon with visible haustrations. The wall thickening and peri-intestinal fatty tissue reaction (hyperechogenic appearance) suggest inflammatory bowel disease. $\mathrm{b}$ The detection of thickening of the jejunal wall (panel b) in the same patient was suggestive of Crohn's disease.

organic lesions of the colon but are not recommended as preferred techniques for investigating patients with suspected or known functional disease like SUDD.

Despite the fact that there are no standardized diagnostic criteria for SUDD, some guidelines recommended GIUS as a first-line examination in patients with suspected acute diverticulitis. GIUS has also been used for monitoring patients with acute diverticulitis. It can detect diverticula of the sigmoid colon as protuberances of the colonic wall, containing gaseous or solid artifacts, sometimes associated with acoustic shadowing due to the presence of a fecolith within the diverticular lumen ( $\triangleright$ Fig. 11) with high sensitivity (85\%) and specificity (93\%) [53]. The noninflamed diverticular wall is usually undetectable by IUS, but some changes of the colonic wall (e.g., hypertrophy of the muscularis propria) [53], can be de-

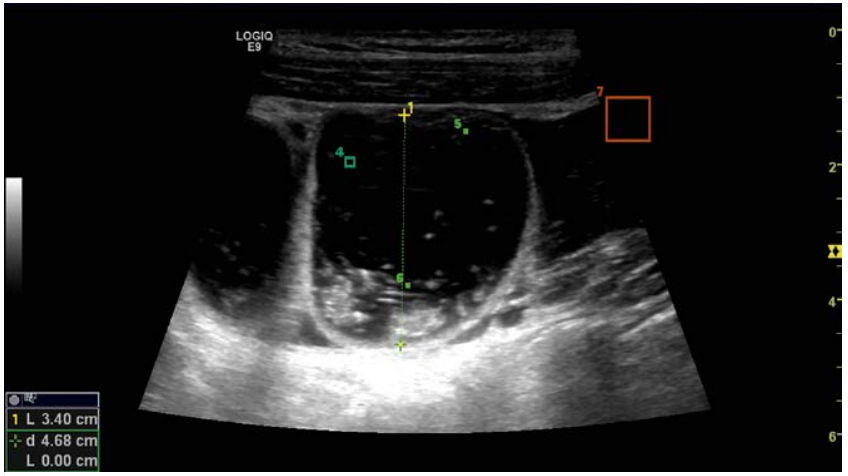

- Fig. 10 Bowel scanning with $9 \mathrm{MHz}$ linear array in small intestinal segments of a patient with GUCY2C syndrome. Familial GUCY2C diarrhea syndrome (FGDS) is a rare inherited disorder with clinical characteristics of irritable bowel syndrome (IBS-D) but also with increased risk of Crohn's disease. Typical findings are an increased number of fluid-filled loops, many of which are pathologically dilated, and dysmotility. This image shows a dilated small intestine (3.4 $\mathrm{cm}$ ) and a magnetic positioning navigation system (dots and boxes) on a GE Logiq E9 was used to count the fluid-filled loops.

tected and be potentially useful in the management of patients with SUDD.

\section{Statement 12}

GIUS may detect diverticula of the sigmoid colon and support the diagnosis of symptomatic uncomplicated diverticular disease of the colon. [LoE 3; GoR 4]

Consensus level of agreement: A+ 10/12, A- 2/12

\section{Chronic constipation}

Constipation is one of the most common digestive problems affecting people of all ages. In chronic constipation due to fecal impaction, GIUS can detect hard stools within the colon as an intraluminal hypoechoic mass with posterior shadowing of varying diameter depending on the site and amount of fecal collection [54]. In children, the detection of hard stools in the rectum with an enlarged rectal diameter has been used as a predictor of constipation and fecal impaction. In particular, a rectopelvic ratio (the width of the rectum/the distance between the iliac spines) greater than 0.189 allowed the detection of megarectum with fecal impaction with a sensitivity of $88.3 \%$ [55]. In healthy adults, fecal retention of the rectum can also be detected by pocketsize US as a rectal area $>4.0 \mathrm{~cm}$ in diameter ( $\triangleright$ Fig. 12) [56].

GIUS has also been proposed to assess the severity of constipation by means of specific scores. One of these, developed in the pediatric population, takes into account the height of the stool and its effect on the bladder with a good correlation with symptoms [57]. Another score [58], proposed for adult patients, assessed the fecal loading and its consistency using CT as the reference standard. Other scores that are rather complex and not yet validated have been proposed to assess bowel frequency and stool consistency in adults. These scores, based on the transverse diameters of the colonic segments the acoustic shadowing of the contents and haustrations appearance [58], showed a good correlation with CT find- 


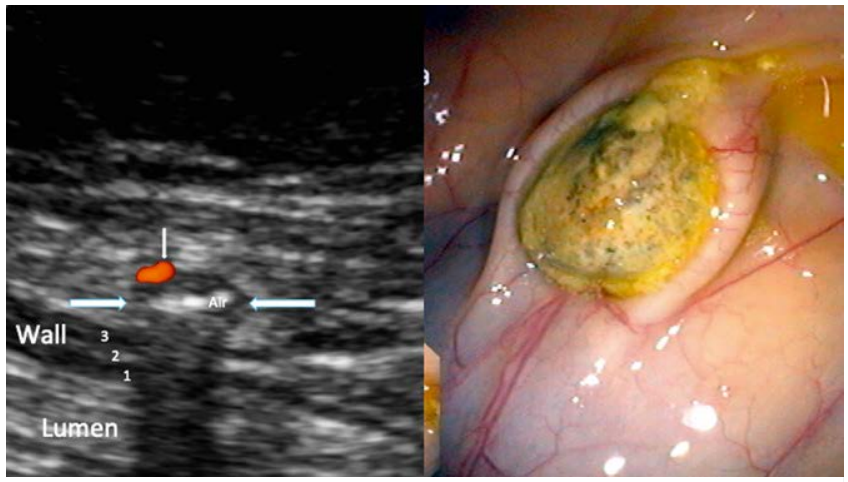

- Fig. 11 In the left panel, a diverticulum (arrows) originating in the left colon is seen with air inside and a small vessel at the periphery. In the right panel, the corresponding endoscopic image is shown with a fecalith entrapped in the diverticulum.

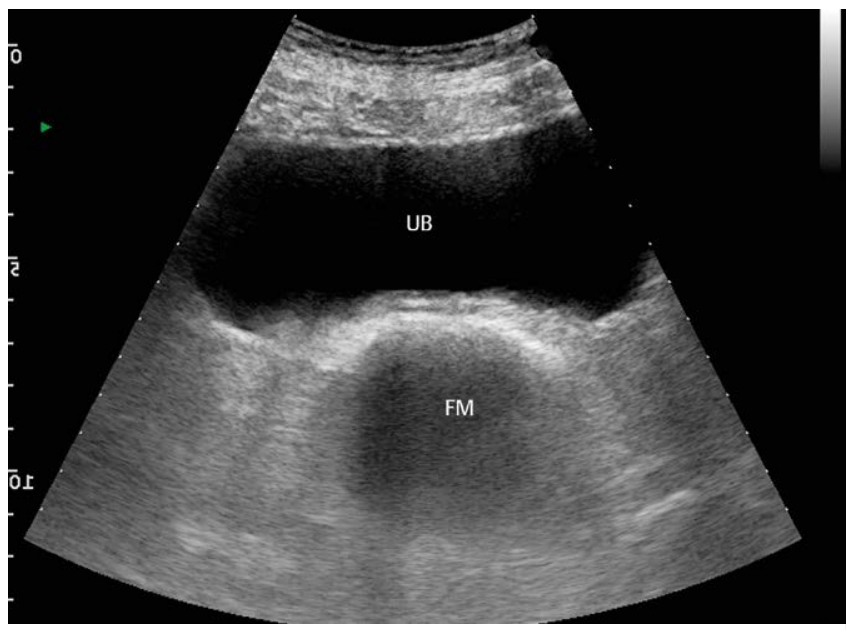

- Fig. 12 This ultrasonogram demonstrates a fecal mass (FM) with a hyperechogenic surface positioned in the rectum posterior to a well-filled urinary bladder (UB) in a patient with obstipation.

ings of stool and/or gas distribution and with the colonic transit time evaluated by radiopaque markers.

\section{Statement 13}

GIUS can be used to detect fecal load in chronic constipation and specifically rectal fecal impaction, particularly in children [LoE 3, GoR 4]

Consensus level of agreement: A+10/12, A- 2/12

\section{Discussion and Conclusion}

The data from the literature show that GIUS may be helpful in patients presenting with symptoms suspicious for FGID for the differentiation from organic diseases and may suggest the underlying pathophysiological mechanisms. This is particularly valid in children and young patients as well as in very old and institutionalized patients where diagnostic examinations are sometimes difficult to perform. In light of the fact that GIUS is widely available, can be quickly applied, is noninvasive, and is cost-effective, it may help to speed up and accurately address the diagnostic workup.

In the absence of organic lesions and knowing some of the functional sonographic features, which involve the motility of the wall and the contents and diameter of the GI lumen, GIUS can help to corroborate the diagnosis of a functional disease. Furthermore, the high temporal and spatial resolution of GIUS make it an optimal tool for patient follow-up and for monitoring the disease and treatment response.

However, the lack of standard sonographic criteria for FGID and the paucity of clinical studies are limitations regarding the use of GIUS in routine clinical practice. For some clinical conditions shown in this review, the level of usefulness of GIUS is still uncertain, as pointed out by the low level of evidence of the statements. However, we believe that the studies on these topics, even though many of them are explorative studies, deserve to be mentioned for the sake of comprehensiveness, to provide information for inexperienced sonographers, and to provide information for those who wish to further investigate these topics with advanced and tailored techniques. In fact, further studies are welcome to identify more pathophysiological sonographic features of specific FGID and to show the effectiveness in clinical trials and practice.

\section{Conflict of Interest}

Giovanni Maconi, Trygve Hausken, Christoph Frank Dietrich, Nadia Pallotta, Ioan Sporea, Dieter Nuernberg, Klaus Dirks, Laura Romanini, Carla Serra, Barbara Braden and Zeno Sparchez have no Conflict of Interest to declare with this manuscript. Odd Helge Gilja has received speaker honoraria from Bracco, GE Healthcare, and Takeda AS.

\section{References}

[1] Ødegaard S, Gilja OH, Gregersen H. Editors of book: Basic and New Aspects of Gastrointestinal Ultrasonography. 2005: 1-502. Publisher: World Scientific; Singapore: ISBN 981-238-845-1

[2] Nylund K, Maconi G, Hollerweger A et al. EFSUMB Recommendations and Guidelines for Gastrointestinal Ultrasound. Ultraschall Med 2017; 38: $273-284$

[3] Hollerweger A, Maconi G, Ripolles T, Nylund K, Higginson A, Serra C et al. Gastrointestinal ultrasound (GIUS) in intestinal emergencies. An EFSUMB position paper. Ultraschall Med 2020, doi: 10.1055/a-1147-1295

[4] Dietrich CF, Hollerweger A, Dirks K et al. EFSUMB Gastrointestinal Ultrasound (GIUS) Task Force Group: Celiac sprue and other rare gastrointestinal diseases ultrasound features. Med Ultrason 2019; 21: 299-315

[5] Nuernberg D, Saftoiu A, Barreiros AP et al. EFSUMB Recommendations for Gastrointestinal Ultrasound Part 3: Endorectal, Endoanal and Perineal Ultrasound. Ultrasound Int Open 2019; 5: E34-E51

[6] Dirks K, Calabrese E, Dietrich CF et al. EFSUMB Position Paper: Recommendations for Gastrointestinal Ultrasound (GIUS) in Acute Appendicitis and Diverticulitis. Ultraschall Med 2019; 40: 163-175

[7] Maconi G, Nylund K, Ripolles T et al. EFSUMB Recommendations and Clinical Guidelines for Intestinal Ultrasound (GIUS) in Inflammatory Bowel Diseases. Ultraschall Med 2018; 39: 304-313

[8] Turley R, Cohen S. Impact of voice and swallowing problems in the elderly. Otolaryngol Head Neck Surg 2009; 140: 33-36 
[9] Blyth KM, McCabe P, Madill C, Ballard KJ. Ultrasound in dysphagia rehabilitation: a novel approach following partial glossectomy. Disabil Rehabil 2017; 39: 2215-2227

[10] Lee YS, Lee KE, Kang Y, Yi TI, Kim JS. Usefulness of Submental Ultrasonographic Evaluation for Dysphagia Patients. Ann Rehabil Med 2016; 40: 197-205

[11] Achille G, Castellana M, Russo S, Montepara M, Giagulli VA, Triggiani V. Zenker Diverticulum: A Potential Pitfall in Thyroid Ultrasound Evaluation: A Case Report and Systematic Review of Literature. Endocr Metab Immune Disord Drug Targets 2019; 19: 95-99

[12] Cui XW, Ignee A, Baum U, Dietrich CF. Feasibility and usefulness of using swallow contrast-enhanced ultrasound to diagnose Zenker's diverticulum: preliminary results. Ultrasound Med Biol 2015; 41: 975-981

[13] Takebayashi S, Matsui K, Ozawa Y, Nozawa T, Fujioka E. Cervical esophageal motility: evaluation with US in progressive systemic sclerosis. Radiology 1991; 179: 389-393.

[14] Zuber-Jerger I, Müller A, Kullmann F, Gelbmann CM, Endlicher E, Müller-Ladner U, Fleck M. Gastrointestinal manifestation of systemic sclerosis--thickening of the upper gastrointestinal wall detected by endoscopic ultrasound is a valid sign. Rheumatology (Oxford) 2010; 49: $368-372$

[15] Nam IC, Choi H, Kim ES, Mo EY, Park YH, Sun DI. Characteristics of thyroid nodules causing globus symptoms. Eur Arch Otorhinolaryngol 2015; 272 (5): 1181-1188

[16] Järvenpää P, Ilmarinen T, Geneid A et al. Work-up of globus: assessing the benefits of neck ultrasound and videofluorography. Eur Arch Otorhinolaryngol 2017; 274: 931-937

[17] Eskander A, Monteiro E, O'Connell D, Taylor SM. Canadian Association of Head and Neck Surgical Oncology (CAHNSO). Head and Neck Surgical Oncology Choosing Wisely Campaign: imaging for patients with hoarseness, fine needle aspiration for neck mass, and ultrasound for odynophagia. J Otolaryngol Head Neck Surg 2018; 47: 2

[18] Eckardt VF, Schmitt T, Kanzler G. Transabdominal ultrasonography in achalasia. Scand J Gastroenterol 2004; 39 (7): 634-647. doi:10.1080/00365520410005478; PMID: 15370683

[19] Minella R, Minelli R, Rossi E, Cremone G, Tozzi A. Gastroesophageal and gastric ultrasound in children: the state of the art. J Ultrasound 2020, doi: 10.1007/s40477-020-00471-w. Epub ahead of print. PMID: 32361921

[20] Savino A, Cecamore C, Matronola MF, Verrotti A, Mohn A, Chiarelli F, Pelliccia P. US in the diagnosis of gastroesophageal reflux in children. Pediatr Radiol. 2012; 42(5):515-524. doi: 10.1007/s00247-0122344-z. Epub 2012 Mar 9. PMID: 22402830

[21] Barone M, Di Lernia P, Carbonara M, Ladisa R, Donno A, Amoruso A, Di Leo A, Francavilla A. Sliding gastric hiatal hernia diagnosis by transabdominal ultrasonography: an easy, reliable and non-invasive procedure. Scand J Gastroenterol 2006; 41 (7): 851-855. doi: 10.1080/00365520500453408. PMID: 16785200

[22] Gilja OH. Ultrasound of the stomach--the EUROSON lecture 2006. Ultraschall Med 2007; 28: 32-39

[23] Gilja OH, Hatlebakk JG, Odegaard S et al. Advanced imaging and visualization in gastrointestinal disorders. World J Gastroenterol 2007; 13: $1408-1421$

[24] Pallotta N, Cicala M, Frandina C, Corazziari E. Antro-pyloric Contractile Patterns and Transpyloric Flow After Meal Ingestion in Humans. Am J Gastroenterol 1998; 93: 2513-2522

[25] Hveem K, Sun WM, Hebbard G, Horowitz M, Doran S, Dent J. Relationship between ultrasonically detected phasic antral contractions and antral pressure. Am J Physiol Gastrointest Liver Physiol 2001; 281: G95-G101
[26] Savoye-Collet C, Savoye G, Smout A. Determinants of transpyloric fluid transport: a study using combined real-time ultrasound, manometry, and impedance recording. Am J Physiol Gastrointest Liver Physiol 2003; 285 (6): G1147-G1152

[27] Hausken T, Mundt M, Samsom M. Low antroduodenal pressure gradients are responsible for gastric emptying of a low-caloric liquid meal in humans. Neurogastroenterol Motil 2002; 14 (1): 97-105

[28] Hveem K, Svebak S, Hausken T, Berstad A. Effect of mental stress and cisapride on autonomic nerve functions in functional dyspepsia. Scand J Gastroenterol 1998; 33: 123-127

[29] Ahluwalia NK, Thompson DG, Mamtora H, Hindle J. Evaluation of gastric antral motor performance in patients with dysmotility-like dyspepsia using real-time high-resolution ultrasound. Neurogastroenterol Motil 1996; 8: 333-338

[30] Gilja $\mathrm{OH}$, Heimdal A, Hausken T et al. Strain during gastric contractions can be measured using Doppler Ultrasonography. Ultrasound Med Biol 2002; 28: 1457-1465

[31] Ahmed AB, Matre K, Hausken T, Gregersen H, Gilja OH. Rome III subgroups of functional dyspepsia exhibit different characteristics of antral contractions measured by strain rate imaging - a pilot study. Ultraschall Med 2012; 33: E233-40

[32] Bolondi L, Bortolotti M, Santi V, Calletti T, Gaiani S, Labo G. Measurement of gastric emptying time by real-time ultrasonography. Gastroenterology 1985; 89: 752-759

[33] Gentilcore D, Hausken T, Horowitz M, Jones KL. Measurements of gastric emptying of low- and high-nutrient liquids using 3D ultrasonography and scintigraphy in healthy subjects. Neurogastroenterol Motil 2006; 18: 1062-1068

[34] Duan LP, Zheng ZT, Li YN. A study of gastric emptying in non-ulcer dyspepsia using a new ultrasonographic method. Scand J Gastroenterol 1993; 28: 355-360

[35] Sogabe M, Okahisa T, Tsujigami K et al. Ultrasonographic assessment of gastric motility in diabetic gastroparesis before and after attaining glycemic control. J Gastroenterol 2005; 40: 583-590

[36] Hausken T, Gilja OH, Undeland KA, Berstad A. Timing of postprandial dyspeptic symptoms and transpyloric passage of gastric contents. Scand J Gastroenterol 1998; 33: 822-827

[37] Gilja OH, Lunding J, Hausken T, Gregersen H. Gastric accommodation assessed by ultrasonography. World J Gastroenterol 2006; 12 : 2825-2829

[38] Gilja OH, Hausken T, Wilhelmsen I, Berstad A. Impaired accommodation of proximal stomach to a meal in functional dyspepsia. Dig Dis Sci 1996; 41: 689-696

[39] Steinsvik EK, Hausken T, Gilja OH. The ultrasound meal accommodation test in 509 patients with functional gastrointestinal disorders. Scand J Gastroenterol 2016; 51: 788-794

[40] Storlid EL, Hausken T, Lied GA, Gilja OH, Hatlebakk JG. Gastric accommodation in healthy subjects studied by ultrasound, manometry, and impedancemetry. Neurogastroenterol Motil 2018; 30: e13249

[41] Gilja OH, Thune N, Matre K, Hausken T, Odegaard S, Berstad A. In vitro evaluation of three-dimensional ultrasonography in volume estimation of abdominal organs. Ultrasound Med Biol 1994; 20: 157-165

[42] Gilja OH, Detmer PR, Jong JM et al. Intragastric distribution and gastric emptying assessed by three-dimensional ultrasonography. Gastroenterology 1997; 113: 38-49

[43] Steinsvik EK, Valeur J, Hausken T, Gilja OH. Postprandial Symptoms in Patients With Functional Dyspepsia and Irritable Bowel Syndrome: Relations to Ultrasound Measurements and Psychological Factors. J Neurogastroenterol Motil 2020; 26: 96-105

[44] Gilja OH, Heimdal A, Hausken T et al. Strain during gastric contractions can be measured using Doppler ultrasonography. Ultrasound Med Biol 2002; 28: 1457-1465 
[45] Soncini M, Stasi C, Usai Satta P et al. AIGO. IBS clinical management in Italy: The AIGO survey. Dig Liver Dis 2019; 51: 782-789

[46] Francis CY, Duffy JN, Whorwell PJ, Martin DF. Does routine abdominal ultrasound enhance diagnostic accuracy in irritable bowel syndrome? Am J Gastroenterol 1996; 91: 1348-1350

[47] Dilillo D, Zuccotti GV, Galli E et al. Noninvasive testing in the management of children with suspected inflammatory bowel disease. Scand J Gastroenterol 2019; 54: 586-591

[48] Novak KL, Jacob D, Kaplan GG, Boyce E, Ghosh S, Ma I, Lu C, Wilson S, Panaccione R. Point of Care Ultrasound Accurately Distinguishes Inflammatory from Noninflammatory Disease in Patients Presenting with Abdominal Pain and Diarrhea. Can J Gastroenterol Hepatol 2016; 2016: 4023065

[49] O'Connor O], McSweeney SE, McWilliams S, O'Neill S, Shanahan F, Quigley EM, Maher MM. Role of radiologic imaging in irritable bowel syndrome: evidence-based review. Radiology. 2012; 262 (2): 485-494. doi: 10.1148/radiol.11110423. Epub 2011 Dec 9. PMID: 22156992

[50] Crade M, Pham V. Ultrasound examination of the sigmoid colon: possible new diagnostic tool for irritable bowel syndrome. Ultrasound Obstet Gynecol 2006; 27: 206-209

[51] Smereczyński A, Starzyńska T, Kołaczyk K. Ultrasound of selected pathologies of the small intestine. J Ultrason 2013; 13: 155-166
[52] von Volkmann HL, Nylund K, Tronstad RR et al. An activating gucy2c mutation causes impaired contractility and fluid stagnation in the small bowel. Scand J Gastroenterol 2016; 1-8

[53] Hollerweger A, Macheiner P, Hubner E et al. Colonic diverticulosis: a comparison between sonography and endoscopy. Ultraschall Med 2002; $231: 41-46$

[54] Derchi LE, Musante F, Biggi E, Cicio GR, Oliva L. Sonographic appearance of fecal masses. J Ultrasound Med 1985; 4: 573-575

[55] Bijoś A, Czerwionka-Szaflarska M, Mazur A, Romañczuk W. The usefulness of ultrasound examination of the bowel as a method of assessment of functional chronic constipation in children. Pediatr Radiol 2007; 37: 1247-1252

[56] Yabunaka K, Matsumoto M, Yoshida M et al. Assessment of rectal feces storage condition by a point-of-care pocket-size ultrasound device for healthy adult subjects: A preliminary study. Drug Discov Ther 2018; 12: $42-46$

[57] Lakshminarayanan B, Kufeji D, Clayden G. A new ultrasound scoring system for assessing the severity of constipation in children. Pediatr Surg Int 2008; 24: 1379-1384

[58] Yabunaka K, Matsuo J, Hara A et al. Sonographic Visualization of Fecal Loading in Adults: Comparison With Computed Tomography. Journal of Diagnostic Medical Sonography 2015; 31: 86-92 